\title{
Prevalencia de Bartonella henselae en donantes de sangre y riesgo de transmisión sanguínea en Chile
}

\author{
M. Antonieta Núñez, Karla Contreras, M. Soledad Depix, Enrique Geoffroy, \\ Nicolás Villagra, Sandra Mellado y Ana M. Salinas
}

\section{Prevalence of Bartonella henselae in blood donors and risk of blood transmission in Chile}

Background: Bartonella henselae is the causal agent of cat scratch disease in immunocompetent persons and bacterial angiomatosis in immunocompromised patients. In Chile, the prevalence of antibodies against $B$. henselae in healthy children and adolescents is $13.3 \%$, in persons with occupational risk $60.5 \%$, and in cats $85.6 \%$. There are no published data regarding the seroprevalence in blood donors in our country, so determining if $B$. henselae is present in the blood of donors at the time of donation is very important, since this microorganism can survive up to 35 days in the red blood cells stored in a blood bank at $4{ }^{\circ} \mathrm{C}$. Objective: To determine the prevalence of $B$. henselae in blood donors. Methodology: 140 donor blood samples were analyzed to detect the presence of $B$. henselae, using the polymerase chain reaction technique. Results: $13.6 \%$ of the blood donors with positive polymerase chain reaction for $B$. henselae were obtained. The sequence of the amplified fragments showed an identity of over $98 \%$ with respect to $B$. henselae reference sequences. Conclusion: The risk of blood transmission is due to a country with high $B$. henselae infection.

Key words: Bartonella henselae, blood donors, transfusion.

Palabras clave: Bartonella henselae, donantes de sangre, transfusión.

\section{Introducción}

$B$ artonella henselae es un bacilo gramnegativo, intraeritrocitario que requiere medios enriquecidos y $5 \%$ de $\mathrm{CO}_{2}$ para crecer, por lo que es considerado como un microorganismo fastidioso ${ }^{1,2}$.

Este es un patógeno zoonótico de distribución universal, que tiene como reservorio animal al gato. La transmisión entre ellos se produce a través del vector artrópodo o pulga de los gatos infectados (Ctenocephalides felis) y la transmisión accidental al humano ocurre por arañazos o mordeduras de gatos infectados, y a través de la picadura que producen las pulgas infectadas ${ }^{1,2}$.

Al transmitirse esta bacteria al humano, el ciclo de infección se inicia con la colonización del endotelio vascular. Aproximadamente en el quinto día de infección, las bacterias ingresan de forma sincrónica desde el endotelio vascular hacia el torrente sanguíneo, donde infectan a los eritrocitos de manera eficiente, permaneciendo dentro de ellos en un estado no replicativo por varias semanas ${ }^{2-4}$.

La infección humana por $B$. henselae puede ocasionar una amplia gama de síntomas clínicos, los que dependen del estado inmunológico del hospedero. En pacientes inmunocompetentes, la manifestación más frecuente es la enfermedad del arañazo de gato, y en los pacientes inmunocomprometidos la infección se caracteriza por presentar: fiebre de origen desconocido, endocarditis con cultivo negativo, o lesiones angioproliferativas que podrían afectar virtualmente a cualquier órgano, pero tiene predilección por la piel, hígado y bazo ${ }^{4-6}$.

En nuestro país la infección por $B$. henselae es endémica en gatos con una seroprevalencia de $85,6 \% 7$.

En un estudio realizado recientemente en personas con riesgo ocupacional (veterinarios y cuidadores de felinos), se detectó una alta seroprevalencia de infección por $B$. henselae alcanzando a $60,5 \%{ }^{8}$. Anteriormente se había reportado $10,3 \%$ para el mismo grupo y $13,5 \%$ de seroprevalencia en niños y adolescentes sanos?.

La seroprevalencia de anticuerpos IgG de $B$. henselae en donantes de sangre se ha descrito en múltiples trabajos. En la provincia de Aydin en Turquía se encontró 3,3\% (n: 333), en Nueva Zelanda 3,61\% (n: 140), en Suiza 4,4\% (n: 498), en Sicilia, Italia se encontró $11,4 \%$ (n: 122) y en este caso las muestras positivas fueron testeadas también para IgM siendo todas positivas. En un estudio en Tailandia, en que se analizaron 162 muestras de sangre de individuos sanos, mayormente donantes de sangre, se encontró 5,5\% de muestras positivas para IgG y $1,2 \%$ para $\operatorname{IgM}^{10-14}$.

Recientemente, en el centro de sangre más grande del sureste de Brasil se detectó por técnica de reacción de polimerasa en cadena (RPC) $3,2 \%$ (n: 500) de donantes de sangre positivos para $B$. henselae ${ }^{15}$.
Universidad Santo Tomás, Santiago, Chile. Escuela de Tecnología Médica (MAN, KC, MSD, EG, AMS). Universidad Andres Bello, Santiago, Chile.

Laboratorio Patogénesis Molecular y Antimicrobianos (NV). Hospital San José, Santiago, Chile.

Banco de Sangre (SM).

Fuente de financiamiento: Proyecto de investigación interno Universidad Santo Tomás.

Recibido: 4 de mayo de 2017 Aceptado: 22 de noviembre de 2017

Correspondencia a: María Antonieta Núñez Ahumada antonieta.tm@gmail.com 
En nuestro país no existen reportes de seroprevalencia en donantes de sangre, pero en un trabajo realizado anteriormente por nuestro equipo (datos no publicados), encontramos una seroprevalencia de 7,14\% (n: 168) para anticuerpos de tipo IgG.

Se ha visto que $B$. henselae tiene la capacidad para sobrevivir en eritrocitos almacenados a $4{ }^{\circ} \mathrm{C}$ hasta los 35 días que se conservan en banco de sangre, por lo que la probabilidad de transmitir este microorganismo a receptores de transfusiones es posible, lo que representa un riesgo potencial de la transmisión de este patógeno por vía sanguínea, sobre todo en los pacientes poli transfun$\operatorname{didos}^{16}$. No existen reportes de transmisión transfusional de $B$. henselae en humanos, pero si se ha demostrado la transmisión transfusional en ratones ${ }^{17}$.

Dada la alta seroprevalencia de $B$. henselae descrita en distintas poblaciones en nuestro país, la capacidad de este microorganismo para sobrevivir en los eritrocitos y las características patogénicas que podrían ocasionar patologías crónicas graves en receptores de transfusión inmunocomprometidos, el objetivo de este estudio fue determina la prevalencia de $B$. henselae en donantes de sangre.

\section{Metodología}

\section{Población de estudio}

Hombres y mujeres que asistieron a donar sangre al Banco de Sangre de un hospital de la Región Metropolitana.

\section{Población y muestra}

Se seleccionaron aleatoriamente 140 muestras de sangre de donantes que asistieron al Banco de Sangre del Hospital San José, entre marzo y mayo del año 2011 y que firmaron consentimiento informado para participar en el estudio. El estudio contó con la aprobación de la Jefatura del servicio.

\section{Criterios de inclusión}

Donante de sangre aceptado en la entrevista para donar, de acuerdo a los criterios descritos en la Norma General Técnica 146 que regula el procedimiento de atención de donantes del Ministerio de Salud.

\section{Criterios de exclusión}

Muestras lipémicas y/o hemolizadas.

\section{Detección de B. henselae}

Muestras de sangre: Todas las muestras se obtuvieron anticoaguladas con EDTA y con resultados de serología negativos para VIH, virus hepatitis $\mathrm{C}$, virus hepatitis B, Treponema pallidum, Trypanosoma cruzi y HTLV.
Obtención del concentrado globular: Con la finalidad de obtener eritrocitos concentrados, todas las muestras de sangre se centrifugaron por $10 \mathrm{~min}$ a $4.000 \mathrm{rpm}$, eliminando el plasma sobrenadante. De cada muestra de eritrocitos concentrados se extrajo $100 \mu \mathrm{l}$ para formar pooles de concentrado globular de las distintas muestras de los donantes.

Formación de pooles de muestras de sangre de los donantes: Se formaron 35 pooles, cada pool contenía cuatro muestras de $100 \mathrm{ul}$ del concentrado de eritrocitos, obtenidos de las muestras de sangre de los donantes.

Extracción de ADN. La extracción de ADN de cada pool de muestras se realizó con el kit Qiagen QIAamp DNA Blood Mini Kit, de acuerdo a las indicaciones del fabricante y se utilizó $2 \mu 1$ de ADN para la primera RPC.

Detección de B. henselae por RPC: Se realizó una RPC anidada, para aumentar la sensibilidad analítica y determinar la especie de Bartonella. Se utilizó $0,3 \mu \mathrm{M}$ de cada partidor CAT1 (5'GATTCAATTGGTTTGAAGGAGGCT3') y CAT2 (5'TCACATCACCAGGACGTATTC $\left.3^{\prime}\right)$, específicos para $B$. henselae y $B$. quintana, que amplifican un fragmento de $414 \mathrm{pb}$ del gen $h$ tr $A^{18,19}$.

En la segunda amplificación se utilizó $1 \mu 1$ del ADN amplificado en la primera RPC y $0,1 \mu \mathrm{M}$ cada partidor RH1 (5'GGTGCGTTAATTACCGATCC 3') y CAT2; estos partidores amplifican un fragmento de $390 \mathrm{pb}$ del gen $h$ tr $A$ específico para $B$. henselae ${ }^{18,19}$.

Como control interno en el primer ciclo de amplificación se utilizó la detección del gen betaglobina con 0,2 $\mu \mathrm{M}$ de partidores forward (CAA CTT CAT CCA CGT TCA CC) y reverse (GAA GAG CAA AGG ACA GGT $\mathrm{AC})$, que amplifican un fragmento de $110 \mathrm{pb}^{20}$.

En ambas amplificaciones se utilizaron dos controles positivos, una muestra clínica proveniente de un paciente diagnosticado por clínica como enfermedad del arañazo del gato y con inmunofluorescencia indirecta positiva para Ac IgM e IgG (Focus Diagnostics). El otro control fue ADN de una cepa ATCC 49882 de $B$. henselae HOUSTON-1. Además, se incorporó un control de especificidad de especie de Bartonella, que correspondía a una cepa de $B$. quintana.

La RPC se realizó en el termociclador MyGene Series Peltier Thermal Cycler Model MG96 + con una denaturación inicial a $95^{\circ} \mathrm{C}$ por $4 \mathrm{~min}$, seguido por 35 ciclos de denaturación a $95^{\circ} \mathrm{C}$ por 30 segundos, hibridación a $57^{\circ} \mathrm{C}$ por 30 segundos y elongación a $72{ }^{\circ} \mathrm{C}$ por 30 segundos; finalmente, una extensión a $72{ }^{\circ} \mathrm{C}$ por $7 \mathrm{~min}$. Posteriormente se realizó una electroforesis en gel de agarosa al $2 \%$ teñido con bromuro de etidio. Como marcador de peso molecular se utilizó un ADN ladder (INVITROGEN) de 100 pb. La presencia de una banda de 414 pb se consideró positiva para $B$. henselae o $B$. quintana y la visualización de un fragmento de 110 pb indicó la amplificación del control interno de betaglobina. 
Para la segunda amplificación (RPC anidada), el programa del termociclador fue idéntico a la primera amplificación, sólo que la hibridación fue a $63{ }^{\circ} \mathrm{C}$ por 30 segundos. La electroforesis se realizó en idénticas condiciones que la primera amplificación. La presencia de una banda de 390 pb se consideró positiva para $B$. henselae.

Determinación de la sensibilidad analitica: Para determinar la sensibilidad analítica de la técnica RPC anidada, se extrajo el ADN de la cepa ATCC 49882 de $B$. henselae HOUSTON-1 con el kit QIAGEN-QIAamp DNA y posteriormente se cuantificó en el equipo Nanodrop, determinando una concentración de 13,7 ng/ $\mu$ l. Se realizaron diluciones seriadas $1 / 10$ desde la concentración $13,7 \mathrm{ng} / \mathrm{ul}$ hasta una concentración de $1,37 \mathrm{fg} / \mu 1$.

Detección de B. henselae en muestras de pooles positivos: Para determinar qué muestra era positivas, a cada una de las muestras que constituían un pool con resultado positivo se les realizó la extracción de ADN y amplificación por RPC anidada.

Secuenciación: Para confirmar los resultados obtenidos, se secuenció el gen htrA de los productos amplificados de las muestras positivas en Macrogen Service Sequencing, USA y se realizó el análisis de las secuencias con el programa bioinformático BlastN $\left(\mathrm{N}^{\circ}\right.$ de acceso KU179420.1/KF582603.1).

\section{Resultados}

De los 35 pooles analizados (140 muestras totales), en la primera RPC se obtuvo un pool que amplificó el fragmento de $414 \mathrm{pb}$ del gen $h t r A$ positivo para $B$. henselae/B. quintana. En todos los pooles se obtuvo amplificación de un fragmento de 110 pb del control interno, lo que indica que no hubo presencia de inhibidores en las muestras de sangre. En la RPC anidada, 12 de los 35 pooles (incluyendo el pool que amplificó en la primera RPC), fueron positivos, amplificando un fragmento de $390 \mathrm{pb}$, especifico de B. henselae (Figura 1).

Con la finalidad de identificar cuál de las cuatro muestras que conformaban los 12 pooles, eran positivas, se amplificó cada una de ellas. En la RPC anidada, 19 muestras presentaron amplificación especifica de $B$. henselae. La sensibilidad analítica de esta RPC fue de 1,4 fg/ul. En la Figura 2 se muestra un ejemplo de la amplificación del fragmento de $390 \mathrm{pb}$ correspondiente al gen $h \operatorname{tr} A$.

Para confirmar la especificidad bacteriana, se secuenciaron 18 de las 19 muestras amplificadas. El resultado de la secuenciación de los fragmentos amplificados mostró mayor $98 \%$ de identidad con las secuencias de referencia de $B$. henselae.

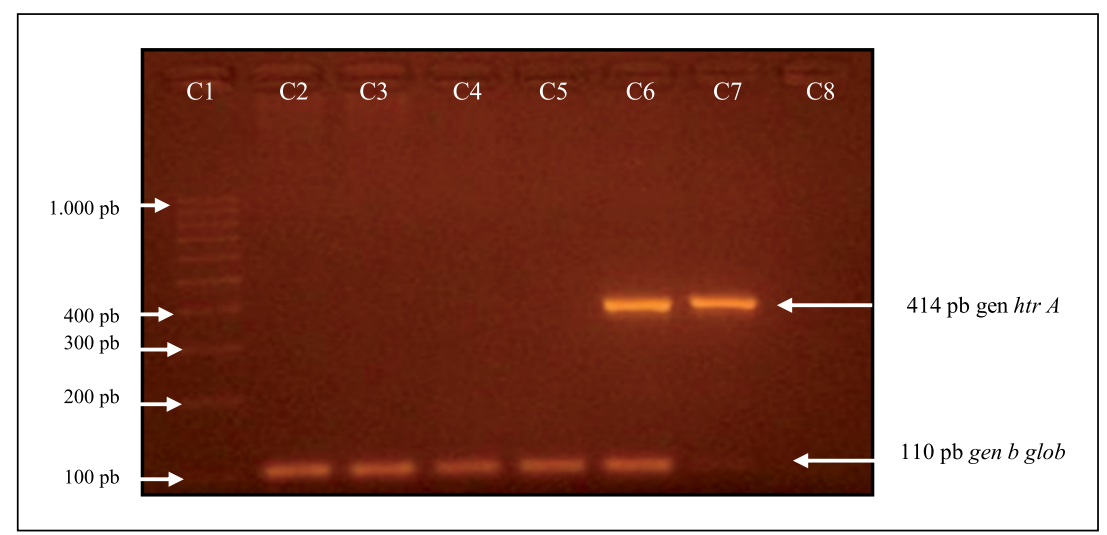

Figura 1. Amplificación del fragmento de $414 \mathrm{pb}$ en muestra individual. Carril 1: ADN ladder 100 pb; Carril 2: muestra 1.868 pool 25; Carril 3: muestra 1.870 pool 25; Carril 4: muestra 1.871 pool 25; Carril 5: muestra 1.872 pool 25; Carril 6: muestra 1.873 pool 26; Carril 7: control positivo de cepa ATCC 49.882 de Bartonella henselae; Carril 8: control negativo.

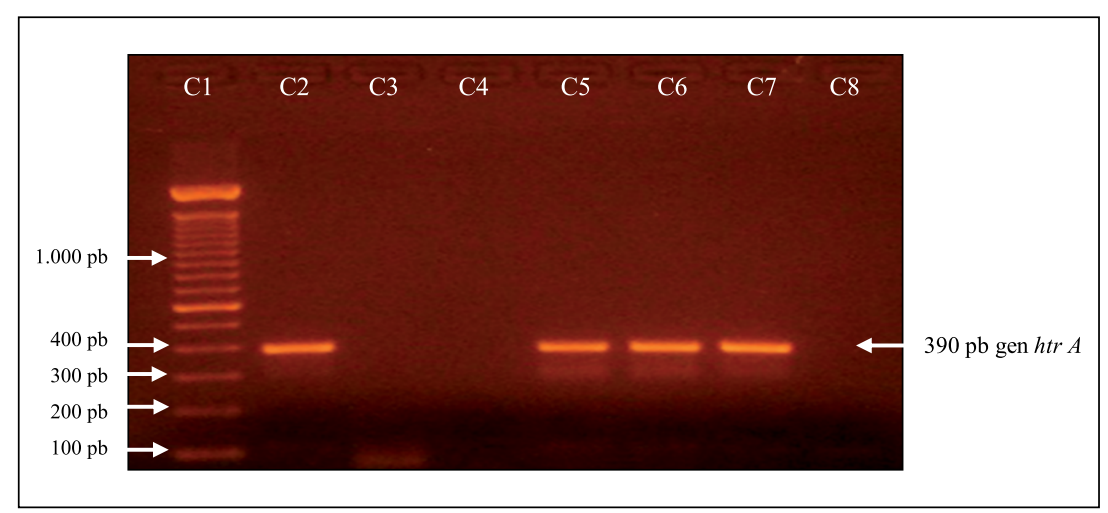

Figura 2. Amplificación del fragmento de 390 pb en muestras individual. Carril 1: ADN ladder 100 pb; Carril 2: muestra 1.972 pool 20; Carril 3: muestra 1.973 pool 20; Carril 4: muestra 1.974 pool 21; Carril 5: muestra 1.795 pool 21; Carril 6: muestra 1.796 pool 21; Carril 7: muestra 1.797 pool 21; Carril 8: muestra 1.868 pool 25.

La prevalencia de $B$. henselae en donantes de sangre, del Hospital San José, fue de 13,6\%. (19/140).

\section{Discusión}

La vigilancia de microorganismos emergentes de relevancia en la seguridad de la transfusión de sangre, es crucial para evitar transmisión de agentes infecciosos que aumentan la morbilidad y mortalidad en pacientes que requieren de terapia transfusional.

En los últimos 20 años, la transmisión de agentes infecciosos por transfusión de productos sanguíneos ha disminuido considerablemente con el aumento de la sensibilidad y especificidad de las técnicas de tamizaje; sin embargo, las técnicas de detección utilizadas se realizan para virus de transmisión transfusional, no siendo obligatorio la detección de la contaminación bacteriana 
al $100 \%$ de las unidades de sangre procesadas. En Chile existe la obligatoriedad de realizar estudios microbiológicos para: VIH, virus de la hepatitis $\mathrm{C}$, virus de la hepatitis B, Treponema pallidum, Trypanosoma cruzi y HTLV I y II, a todas las unidades de sangre que se utilizarán para transfusiones, dejando fuera otros microorganismos ${ }^{21-23}$.

La contaminación bacteriana de unidades de sangre se produce principalmente por dos motivos: bacteriemia asintomática en el donante y contaminación de la unidad con la microbiota habitual de la piel en la flebotomía. En el caso de la contaminación de los hemocomponentes con $B$. henselae, ésta se produce por un donante de sangre con bacteriemia asintomática ${ }^{21}$.

La pesquisa de una unidad de sangre contaminada con $B$. henselae presenta grandes dificultades debido a las características de crecimiento complejas de este microorganismo, por lo que es posible transfundir una unidad contaminada sin que la bacteria haya sido pesquisada, lo que sumado a que la bacteria es viable en los eritrocitos almacenados a $4{ }^{\circ} \mathrm{C}$ en los Bancos de Sangre durante 35 días, genera un alto riesgo de transmisión por transfusión sanguínea ${ }^{1,16}$.

Lo anterior cobra aún mayor relevancia con el antecedente de que se ha encontrado en Chile una alta seroprevalencia de $B$. henselae en gatos, los que siendo portadores transmiten accidentalmente la bacteria al humano por mordeduras o arañazos, indicando una importante fuente de contagio para los potenciales donantes de sangre ${ }^{7}$.

Por otra parte, considerando las diversas patologías que puede producir $B$. henselae, especialmente en pacientes inmunodeprimidos, frecuentes receptores de transfusiones de hemocomponentes, y la alta prevalencia encontrada, podemos sugerir que $B$. henselae es un agente a tener en cuenta en pacientes con neutropenias febriles de origen desconocido que tienen el antecedente de haber recibido transfusión con concentrados de eritrocitos.

La RPC representa una excelente opción para la detección de bacterias fastidiosas que infectan el torrente sanguíneo y que son difíciles de diagnosticar por los métodos tradicionales, como el hemocultivo. Los métodos moleculares reducen el tiempo de detección, aumentan la sensibilidad y especificidad y evitan resultados falsos negativos y positivos, contribuyendo a la identificación de bacterias exigentes e instauración de terapia empírica ${ }^{24}$. Utilizar pooles es una estrategia factible de realizar para la detección $B$. henselae en muestras de donantes de sangre, debido al gran número de muestras que se procesan diariamente y el tiempo de respuesta que se requiere para el diagnóstico ${ }^{25}$.
La comparación entre la primera y segunda amplificación demuestra el alto nivel de sensibilidad de la RPC anidada y refuerza el motivo por el cual se utiliza esta variante de la RPC, en este estudio ${ }^{18,19}$.

Si bien no se realizó un ensayo de sensibilidad analítica con una matriz de sangre, el estudio de sensibilidad analítica con ADN puro de la cepa ATCC de $B$. henselae mostró una excelente sensibilidad logrando una señal de amplificación hasta los 1,4 fg/ul.

Es necesario continuar con más estudios utilizando técnicas aún más sensibles como la RPC en tiempo real, para comprobar la transmisión transfusional de $B$. henselae y el impacto clínico de esta transmisión.

Este es el primer reporte que detecta $B$. henselae en donantes al momento de la donación, en nuestro país, obteniéndose una prevalencia de $13,6 \%$ y dado que este microorganismo puede sobrevivir 35 días a $4{ }^{\circ} \mathrm{C}$, podría existir un riego potencial de transmisión vía sanguínea en Chile, un país con alta seroprevalencia de infección por $B$. henselae.

\section{Resumen}

Introducción: Bartonella henselae es el agente causal de la enfermedad del arañazo del gato en personas inmunocompetentes y de la angiomatosis bacilar y peliosis hepatis en inmunocomprometidos. En Chile la prevalencia de anticuerpos contra $B$. henselae en niños y adolescentes sanos es de $13,3 \%$, en personas con riesgo ocupacional $60,5 \%$ y en gatos $85,6 \%$. No existen datos publicados respecto de la seroprevalencia en donantes de sangre en nuestro país, por lo que determinar si $B$. henselae se encuentra presente en la sangre de los donantes al momento de la donación es muy importante, ya que este microorganismo puede sobrevivir hasta 35 días en los eritrocitos almacenados en banco de sangre a $4{ }^{\circ} \mathrm{C}$. Objetivo: Determinar la prevalencia de $B$. henselae en donantes de sangre. Metodología: Se analizaron 140 muestras de sangre de donantes, para detectar la presencia de $B$. henselae, utilizando la técnica de la reacción de polimerasa en cadena (RPC). Resultados: Se obtuvo $13,6 \%$ de los donantes de sangre con RPC positiva para la $B$. henselae. La secuencia de los fragmentos amplificados presentó una identidad por sobre $98 \%$ con respecto a secuencias de $B$. henselae de referencia. Conclusión: El riesgo de transmisión sanguínea debiera ser considerado en un país con alta seroprevalencia de infección por $B$. henselae. 


\section{Referencias bibliográficas}

1.- Cornejo W, Vizcarra H. Bartonella henselae: Nuevo patógeno en humanos. Anales de la Facultad de Medicina 1999; 60 (4): 281-92.

2.- Versalovic J, Carrol K, Funke G, Jorgensen J, Landry M, Warnock D. Manual of Clinical Microbiology. Vol. 1. Edición10. 2011, cap 46: 786-98.

3.- Pitassi L, Magalhães R F, Barjas-Castro M L, de Paula E V, Ferreira M R, Velho P E. Bartonella henselae infects human erythrocytes. Ultrastructural pathology 2007; 31 (6): 369-72.

4.- Dehio C. Bartonella-host-cell interactions and vascular tumour formation. Nat Rev Microbiol 2005; 3: 621-631.

5.- $\quad$ Mito T, Hirota Y, Suzuki S, Noda K, Uehara T, Ohira Y, et al. Bartonella henselae infective endocarditis detected by a prolonged blood culture. Intern Med 2016; 55 (20): 3065-7.

6.- Arvand M, Schäd S. Isolation of Bartonella henselae DNA from the peripheral blood of a patient with cat scratch disease up to 4 months after the cat scratch injury. J Clin Microbiol 2006; 44: 2288-90.

7.- Ferrés M, Abarca K, Godoy P, García P, Palavecino E, Méndez G, et al. Presencia de Bartonella henselae en gatos: cuantificación del reservorio natural y riesgo de exposición humana a esta zoonosis en Chile. Rev Med Chile 2005; 133: 1465-71.

8.- Troncoso I, Fischer C, Arteaga F, Espinoza C, Azócar, T, Abarca K. Seroprevalence of Bartonella henselae in occupational risk persons. Rev Chilena Infectol 2016; 33 (3): 355.

9.- Ferrés M, Abarca K, Prado P, Montecinos L, Navarrete M, Vial P. Prevalencia de anticuerpos contra Bartonella henselae en niños, en adolescentes y en una población de riesgo ocupacional en Chile. Rev Med Chile 2006;
134: 863-7.

10.- Aydin N, Bülbül R, Tellı M, Gültekın B. Seroprevalence of Bartonella henselae and Bartonella quintana in blood donors in Aydin province, Turkey. Mikrobiyoloji bulteni 2014; 48 (3): 477-83.

11.- Zarkovic A, McMurray C, Deva N, Ghosh S, Whitley D, Guest S. Seropositivity rates for Bartonella henselae, Toxocara canis and Toxoplasma gondii in New Zealand blood donors. Clin Exp Ophthal 2007; 35 (2): 131-4.

12.- Mcgill, S, Wesslén L, Hjelm E, Holmberg M, Auvinen M K, Berggren K, et al. Bartonella spp. seroprevalence in healthy Swedish blood donors. Scand J Infect Dis 2005; 37 (10): 72330.

13.- Mansueto P, Ilenia Pepe, Cillari E, Arcoleo F, Micalizzi A, Bonura F, et al. Prevalence of antibodies anti-Bartonella henselae in Western Sicily: children, blood donors, and cats. J Immun Immunochem 2012; 33 (1): 18-25.

14.- Maruyama S, Boonmar S, Morita Y, Sakai T, Tanaka S. Yamaguchi F, et al. Seroprevalence of Bartonella henselae and Toxoplasma gondii among healthy individuals in Thailand. J Vet Med Sci 2000; 62 (6): 635-7.

15.- De Paiva Diniz P P, Neves Ferreira Velho P E, Urso Pitassi L H, Rovani Drummond M, Bruno Grosselli Lania B, Barjas-Castro M L, et al. Risk factors for Bartonella species infection in blood donors from Southeast Brazil. PLoS Negl Trop Dis 2016; 10 (3): 4509.

16.- Neves Da Silva M, Vieira-Damiani G, Ericson M, Gupta K, Giliolo R, Alemida A, et al. Bartonella henselae transmission by blood transfusion in mice.

17.- Magalhaes R, Pitassi L, Salvadego M, De Moraes A, Barjas-Castro M, Velho P. Bartonella henselae survives after the storage period of red blood cell units: is it transmissible by transfusion? Transfus Med 2008; 18: 28791
18.- Anderson B, Sims K, Regnery R, Robinson L, Schmidt M J, Goral S, et al. Detection of Rochalimaea henselae DNA in specimens from cat scratch disease patients by PCR. J Clin Microbiol 1994; 32: 942-8.

19.- Sander A, Bohm N, Ruess M, Altwegg M. Detection of Bartonella henselae DNA by two different PCR assays and determination of the genotypes of strains involved in histologically defined cat scratch disease. J Clin Microbiol 1999; 37 (4): 993-7.

20.- Guzmán L, Akiba S, Eizuru Y, Castillo D, Corvalan A, Aguayo F. High frequency of p16 promoter methylation in non-small cell lung carcinomas from Chile. Biol Res 2007; 40: 365-72.

21.- Brecher M, Hay SN. Bacterial contamination of blood components. Clini Microbiol Rev 2005; 18 (1): 195-2004.

22.- Norma sobre exámenes microbiológicos obligatorios a realizar a toda la sangre donada para transfusiones y otros aspectos relacionados con la seguridad microbiológica de la sangre. Circular $n^{\circ}$ 53. 1995. Departamento de Epidemiología. División de Programas de Salud. Ministerio de Salud. República de Chile.

23.- American Association of Blood Bank Manual Técnico. Edición 17 ${ }^{a}$. 2011; cap 8: 276-80.

24.- Rutanga JP, Nyirahabimana T. Clinical significance of molecular diagnostic tools for bacterial bloodstream infections: a systematic review. Interdiscip Perspect Infect Dis 2016; 2016: 6412085. doi: 10.1155/2016/6412085.

25.- Roth W, Busch M, Schuller A, Ismay S, Cheng A, Seed C, et al. International survey on NAT testing of blood donations: expanding implementation and yield from 1999 to 2009. Vox Sanguinis 2012; 102 (1): $82-90$. 